\title{
Virtual Flow-Diverter Treatment Planning: The Effect of Device Placement on Bifurcation Aneurysm Haemodynamics
}

\author{
T. W. Peach, K. Spranger and Y. Ventikos
}

\begin{abstract}
Bifurcation aneurysms account for a large fraction of cerebral aneurysms and often present morphologies that render traditional endovascular treatments, such as coiling, challenging and problematic. Flow-diverter stents offer a potentially elegant treatment option for such aneurysms, but clinical use of these devices remains controversial. Specifically, the deployment of a flow-diverter device in a bifurcation entails jailing one or more potentially vital vessels with a low-porosity mesh designed to restrict flow. When multiple device placement configurations exist, the most appropriate clinical decision becomes increasingly opaque.
\end{abstract}

In this study three bifurcation aneurysm geometries were virtually treated by flow-diverter device. Each aneurysm was selected to offer two possible device deployment positions. Flow-diverters similar to commercially available designs were deployed with a fast-deployment algorithm before transient and steady state 
CFD simulations were performed. Reductions in aneurysm inflow, mean- and maximum-WSS, all factors often linked with aneurysm treatment outcome, were compared for different device configurations in each aneurysm.

In each of the three aneurysms modelled, a particular preferential device placement was shown to offer superior performance with the greatest reduction in the flow metrics considered. In all three aneurysm geometries, substantial variations in inflow reduction (up to 25.3\%), mean-WSS reduction (up to 14.6\%), and maximum-WSS reduction (up to $12.1 \%$ ) were seen, which were all attributed to device placement alone. Optimal device placement was found to be non-trivial and highly aneurysm-specific; in only one-third of the simulated geometries, the best overall performance was achieved by deploying a device in the daughter vessel with the highest flow rate.

Good correspondence was seen between transient results and steady state computations that offered a significant reduction in simulation run-time. If accurate steady state computations are combined with the fast-deployment algorithm used, the modest run-time and corresponding hardware make a virtual treatment pipeline in the clinical setting a meaningful possibility. 
Key Words: Cardiovascular Implants, Haemodynamics, Tubes And Flow:

Haemodynamics, Velocity: Haemodynamics, Modelling/ Simulation

[Biomechanics] 


\section{Introduction}

Flow-diverters (FDs) offer a unique and elegant treatment option for cerebral aneurysms at risk of growth or rupture. After a FD device has been deployed, the entire cerebral vessel is effectively reconstructed as the device endothelialises and isolates the aneurysm dome, leading to thrombosis and eventual aneurysm shrinkage and reabsorption.(1) Currently, FDs are recommended for treatment of aneurysms where alternative endovascular (coiling) or surgical (clipping) treatments are unfeasible or have previously failed.(1) However, aneurysms located at vessel bifurcations, which account for the majority of cerebral aneurysms by some estimates,(2) are also thought to be amenable to treatment by FD device. $(3,4)$

Bifurcation aneurysms present a potentially significant, and entirely irreversible, clinical choice when treatment by flow-diverter has been elected: in which daughter vessel should the device be placed and therefore which daughter vessel(s) should be jailed with the device? Such a decision is far from trivial, and a number of competing factors must be weighed. For instance, the haemodynamic environment in and around both treated and untreated bifurcation aneurysms is often highly complex $(5,6)$; small differences in daughter vessel diameter and angle 
may alter the effective length and porosity of the deployed $\operatorname{FD}(7,8)$; reduced flow to jailed daughter vessels may result in loss of vessel patency $(9,10)$; and collateral flow to jailed daughter vessels may prevent aneurysm occlusion.(11) As such, bifurcation aneurysm treatment by FD remains rare and controversial.(12-15)

Modelling of aneurysm treatment, and specifically thrombus genesis and growth, is a highly complex process.(16-18) Although animal models go someway towards capturing the complex biochemistry involved, the aneurysms created in these models are often rudimentary in their morphology and offer little value for patient-specific treatment planning. $(11,19,20)$ Consequently, a number of surrogate measures extracted from patient-specific CFD models are often correlated with likelihood of thrombus growth. Reduction in aneurysm inflow is linked to increased blood residence time in the aneurysm dome and corresponding platelet and fibrin aggregation. $(21,22)$ The role of wall shear stress (WSS) in aneurysm treatment is more complex with extremes of both high- and low-WSS linked to negative outcomes. $(2,5,23,24)$ However, a number of bio-chemical models correlate an overall reduction in WSS with platelet deposition and thrombosis growth. $(25,26)$ But, such heuristics should be viewed cautiously with studies in the literature also suggesting that both spatial and temporal variations 
in WSS distribution, including local jetting and harmonic frequencies, may also play a significant role in thrombus initiation and growth.(27-29)

In this study, three Basilar artery bifurcation aneurysms are examined with CFD models, and the effects of different FD configurations on aneurysm inflow reduction and changes in mean and peak WSS are evaluated. Each aneurysm geometry was chosen to have relatively symmetric-sized daughter vessels (Posterior Cerebral Arteries), thus rendering the treatment decision of where to deploy a FD device non-obvious. 


\section{Methodology}

Three bifurcation aneurysm geometries located at the Basilar tip were selected and segmented from MRA data in OsiriX (OsiriX v.4.1.1, Freeware) and converted to STL format. The geometries were imported into Blender (Stichting Blender Foundation, Amsterdam, The Netherlands) and were trimmed to result in vessel lengths of around five vessel diameters distal and proximal to the aneurysm location, as shown in Figure 1.

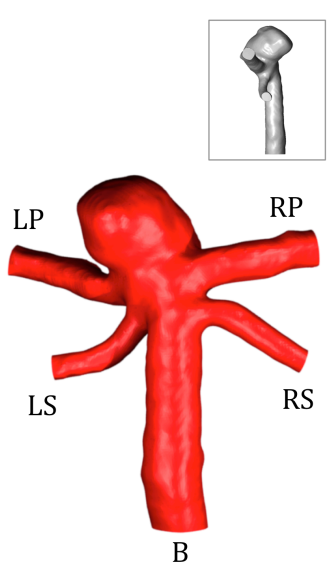

I

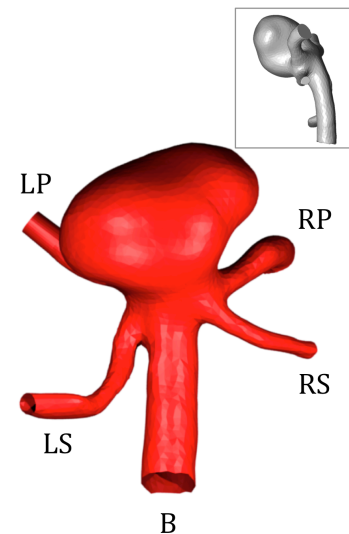

II

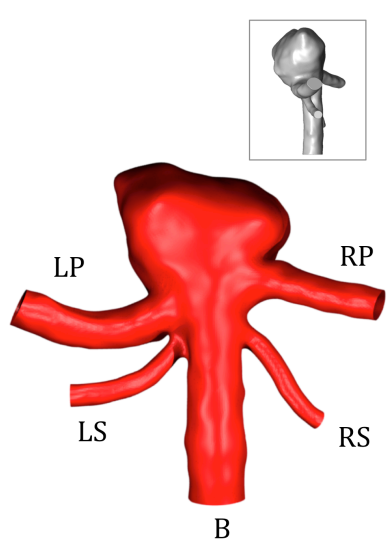

III

Figure 1: Bifurcation aneurysm geometries I, II, and III located at the Basilar tip, with side views inset. (B: Basilar Artery, (L)P: (Left) Posterior Cerebral Artery, (L)S: (Left) Superior Cerebellar Artery) 
A generic flow-diverter device (shown in Figure 2) was created with 70\% porosity and both a mesh design and wire diameter similar to the two devices commonly used clinically: the Silk Flow Diverter (SILK; Balt Extrusion, Montmorency, France) and the Pipeline Embolization Device (PED; Covidien/Medtronic, Minneapolis, MN, USA).

Device Deployment

Device deployment was completed with an in-house fast-deployment algorithm based on a spring analogy and implemented in Matlab (Mathworks, Natick, MA, USA) and Blender, the details of which have been previously reported by the authors $(25,30)$ and are not given here for the sake of brevity. Briefly, the device is first converted to a centreline representation, compressed radially, and aligned with the vessel to mimic the device sheathed by a catheter. The radial compression of the device is then relaxed progressively along its length in an action similar to unsheathing. The device attempts to expand to its stress-free diameter and is opposed by the vessel wall. An offset in the device contact condition then allows for thickness to be added back to the deployed centreline configuration, yielding the 3D device in its deployed position. The deployed device is finally trimmed by 
removing the lengths in the parent and daughter vessels (as shown in Figure 2), in order to reduce the size of subsequently generated CFD meshes, and to improve overall computational efficiency.

\begin{tabular}{|l|l|l|}
\hline II & Left \\
\hline III & & \\
\hline
\end{tabular}


Figure 2: Deployed device positions in each aneurysm geometry (I-III) and each device configuration (Left or Right). Devices are shown trimmed prior to mesh generation

A flow-diverter device was deployed in each aneurysm geometry in two different positions, as shown in Figure 2, corresponding to each PCA. Device equilibrium was achieved in less than 200 iterations of the deployment algorithm and in near real-time. Device sizing was chosen following the SILK and PED manufacturers' guidance.

Meshing and Solver Setup

The aneurysm geometries with and without devices deployed were meshed using CFD-VisCART (ESI Group, Paris, France) using a Projected Single Domain conforming unstructured mesh, an Omnitree Cartesian tree type, and three nearwall Cartesian layers to give a smooth and well-resolved boundary definition. The meshes were then imported into the multi physics suite CFD-ACE+ (ESI Group, Paris, France). 
Blood was modelled as an incompressible fluid with unsteady 3D Navier-Stokes governing equations that were solved following the finite volume approach, with a Central Differencing scheme for spatial differentiations and interpolations, as well as a Crank-Nicholson second-order scheme for time-marching. The SIMPLEConsistent (SIMPLEC) pressure correction method(31,32) and an algebraic multigrid method for convergence acceleration(33) were used. Given previous studies in the literature that confirmed the non-Newtonian effects of blood to be small in the cerebral circulation, $(34,35)$ blood is modelled as a Newtonian fluid with a density of $1000 \mathrm{~kg} / \mathrm{m}^{3}$ and a dynamic viscosity of $0.004 \mathrm{~Pa}$ s. Arterial walls were modelled as rigid, with the effect of such an assumption on flow patterns having been shown to be negligible.(36) A no-slip boundary condition was imposed on both the vessel walls and device struts.

Both steady state and transient computations were performed assuming a mean flow rate of $120 \mathrm{ml} / \mathrm{min}$, a cardiac profile based on that reported by Valencia et al.,(35) and a heart rate of 75 BPM. In both cases, Poiseuille flow was assumed, and a parabolic, radially symmetric velocity profile was prescribed at each geometry inlet along with a constant pressure boundary condition at all outlets. A constant time-step of 0.001 seconds was used across all transient computations with three 
cardiac-cycles simulated. Results were obtained from the third cycle only, to reduce the effects of initial transients.

Inlet Reynolds numbers in the range of 221-270 were seen across the steady state simulations. For the transient simulations, mean inlet Reynolds numbers in the range of 221-270 were observed with an instantaneous value of 192-405. The relatively small inlet Womersley numbers (1.68-1.89) confirmed little departure in transient velocity profile from the parabolic steady state case.

Post-Processing

A plane was placed at the aneurysm neck in each geometry through which aneurysm inflow was measured. This plane also served to separate the aneurysm dome from the parent vasculature with aneurysm WSS then calculated on the aneurysm surface distal of the inlet plane. Transient simulation results were reported as both the mean, calculated by averaging distributions taken at 0.02 seconds intervals, and the range over the cardiac cycle.

Mesh Independence 
The CFD simulations in this study are particularly sensitive to mesh refinement, as the deployed flow-diverter device has a very fine construction $(\sim 35 \mu \mathrm{m}$ wire diameter and $\sim 200 \mu \mathrm{m}$ typical pore size) over which large velocity gradients are present. As such, a comprehensive mesh independence study was performed to evaluate any mesh-induced discretisation error in the key metrics used to evaluate device performance.

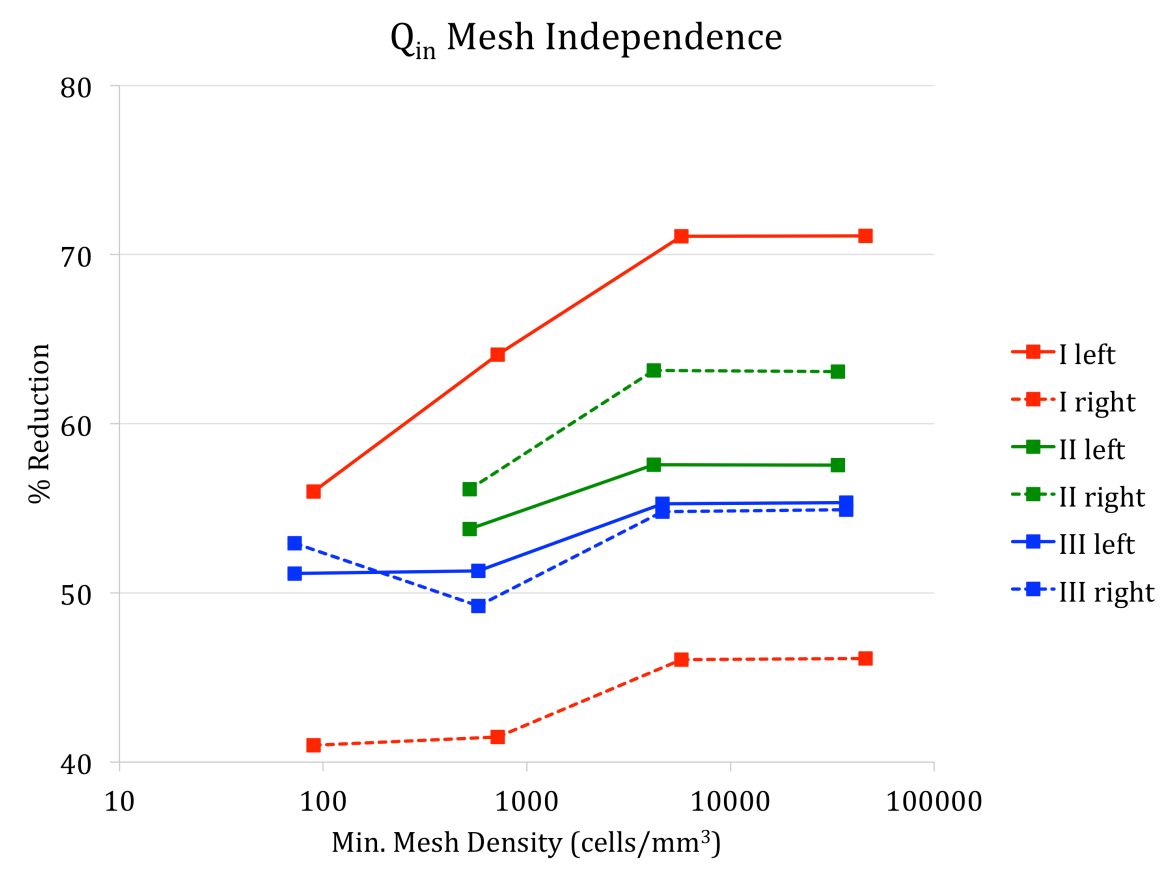

Figure 3: Aneurysm inflow reduction mesh independence showing $<1 \%$ variation in inflow at a minimum mesh density greater than approximately 4,000 cells $/ \mathrm{mm}^{3}$ 
Meshes of all three aneurysm geometries with and without devices deployed were created with approximate minimum mesh densities in the range of 50-50,000 cells $/ \mathrm{mm}^{3}$, which resulted in mesh sizes of $0.088-28.0$ million cells. Steady state computations at mean flow rate were performed for each mesh density across all three geometries using the same solver parameters previously detailed. The relative reduction in aneurysm inflow $\left(\mathrm{Q}_{\text {in }}\right)$, mean aneurysm $\mathrm{WSS}\left(\mathrm{WSS}_{\text {mean }}\right)$, and maximum aneurysm WSS (WSS $\max$ ) for each mesh density in each aneurysm and device combination are detailed in Figures 3, 4 and 5 respectively. 


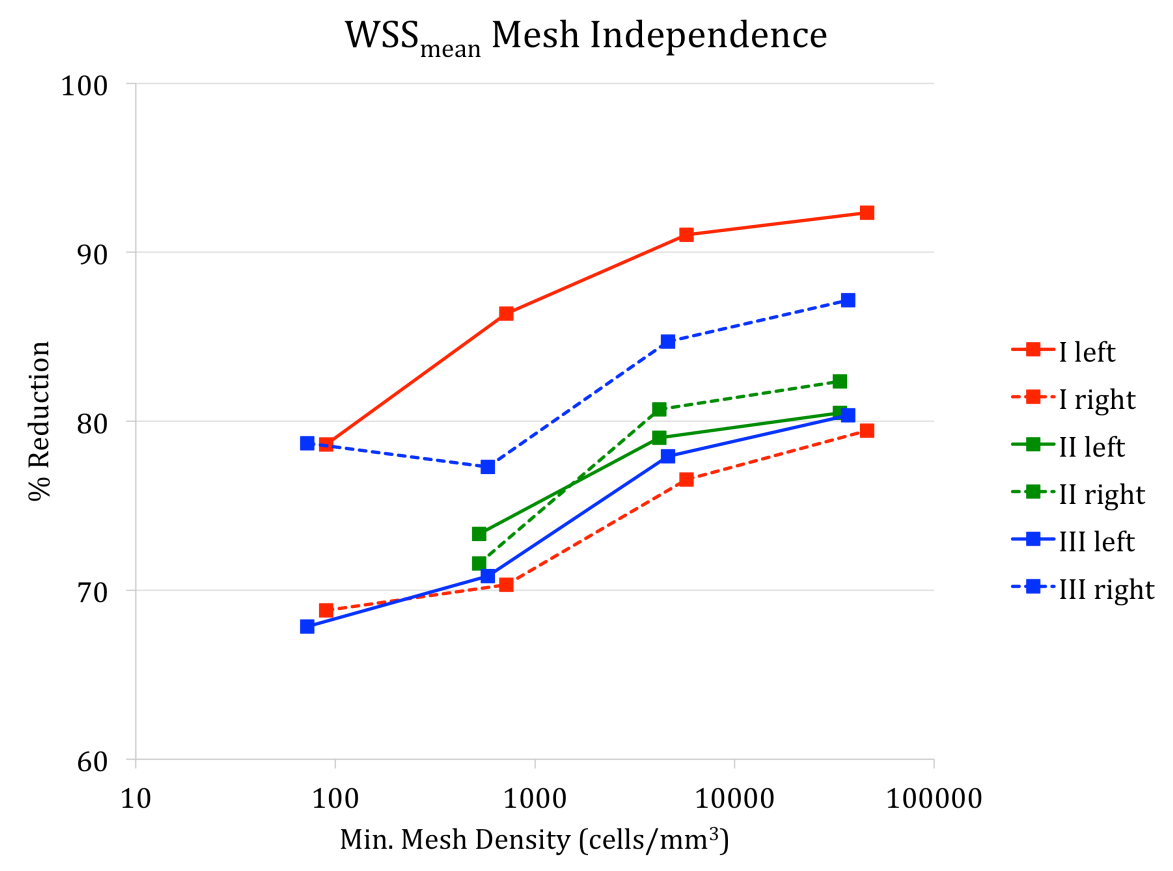

Figure 4: Aneurysm mean WSS reduction mesh independence showing $<2 \%$ variation in inflow at a minimum mesh density greater than approximately 4,000 cells $/ \mathrm{mm}^{3}$

From the figures, it can be assumed that simulations conducted with a minimum mesh density around 4,000 cells $/ \mathrm{mm}^{3}$ result in less than $1 \%$ variation in $Q_{\text {in }}$ and WSS $_{\text {max }}$ and less than $2 \%$ variation in WSS $_{\text {mean, }}$ due to mesh discretisation effects alone. Mesh refinement to this level produced meshes of between 2.88 and 14.7 million cells for the aneurysm geometries with and without a device deployed. Details of the mesh cell size and refinement near the device struts are shown in 
Figure 6 for the level of mesh independence discussed. This level of mesh refinement is consistent with previous mesh independence studies conducted by the authors for both steady state and transient computations.(25)

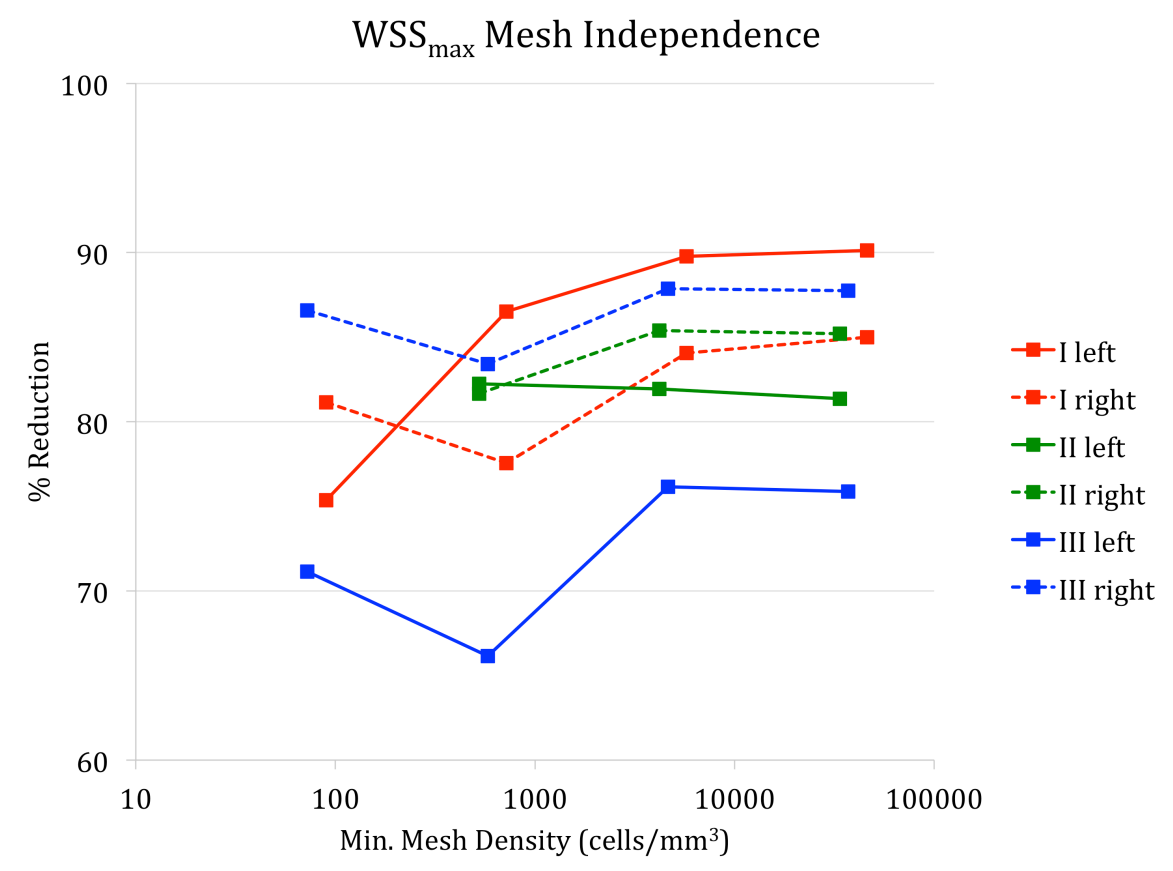

Figure 5: Aneurysm maximum WSS reduction mesh independence showing $<1 \%$ variation in inflow at a minimum mesh density greater than approximately 4,000 cells $/ \mathrm{mm}^{3}$ 
Solution time-step independence for the transient computations was also assumed at a time-step of 0.001 seconds, as previously detailed. No appreciable difference was observed in the metrics discussed above for solutions conducted at time-steps of 0.01-0.001 seconds, in line with similar studies by the authors reported elsewhere.(25) In this particular study the greatest computational efficiency was achieved with the 0.001-second time-step.

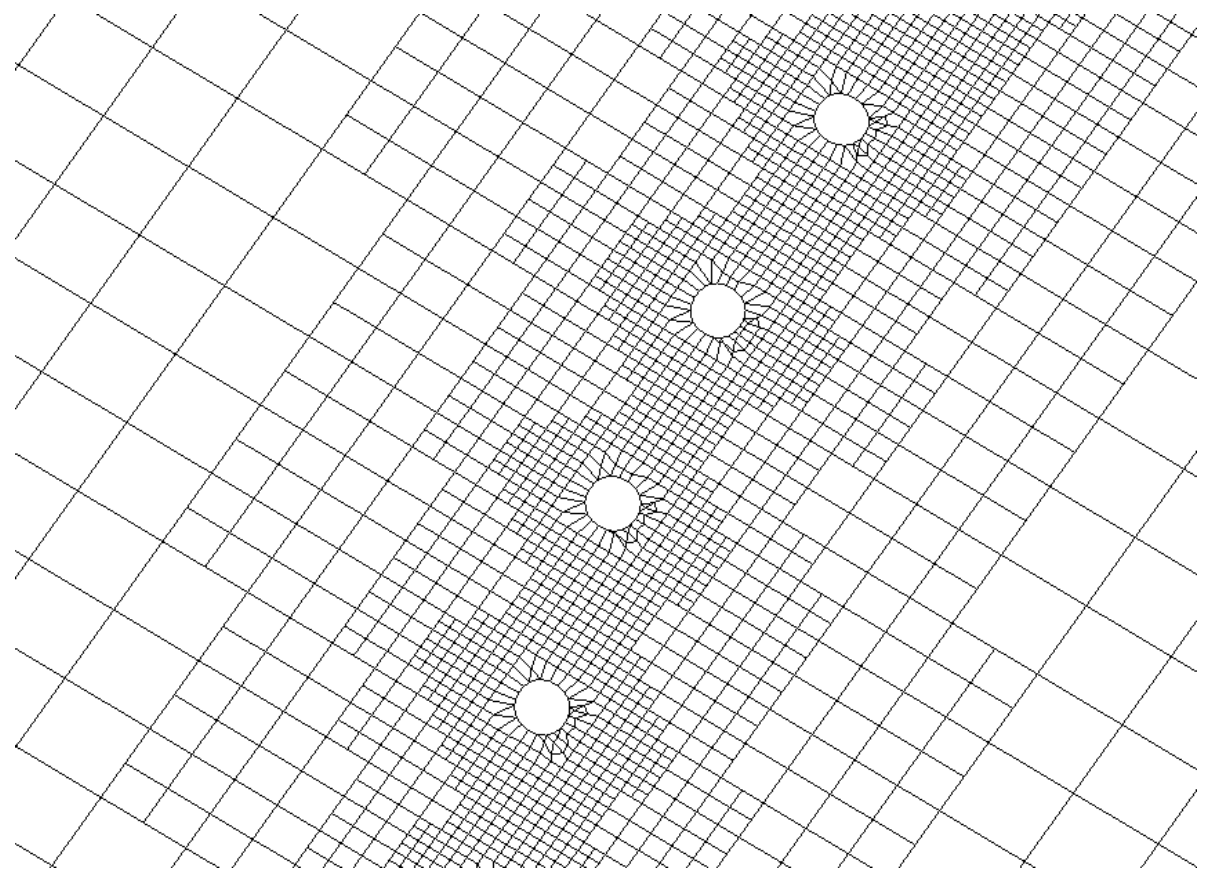

Figure 6: Detail of mesh cell size and refinement at flow-diverter device struts for the "mesh independent" mesh discussed 


\section{Results and Discussion}

The corresponding reductions in aneurysm inflow $\left(\mathrm{Q}_{\text {in }}\right)$, mean WSS $\left(\mathrm{WSS}_{\text {mean }}\right)$ and

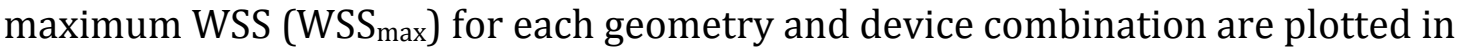
Figures 7, 8 and 9 respectively. Across all three metrics plotted in the figures it is apparent that the steady state solution (indicated in red) offers a good approximation to the mean transient distribution (in blue), despite around a 50fold reduction in computational load.

Transient variation in the reduction of both aneurysm inflow and WSS (indicated in black for Figures 7, 8 and 9) is relatively similar across all aneurysm and device combinations. In all cases, the lowest reduction is seen at maximum parent vessel flow rate (peak systole) and the greatest reduction is seen at minimum parent vessel flow rate. These two conditions correspond to approximately $150 \%$ and $87 \%$ of mean parent vessel flow rate respectively, with a similar degree of asymmetry then seen in the corresponding variation about the mean percentage reduction in each case. 


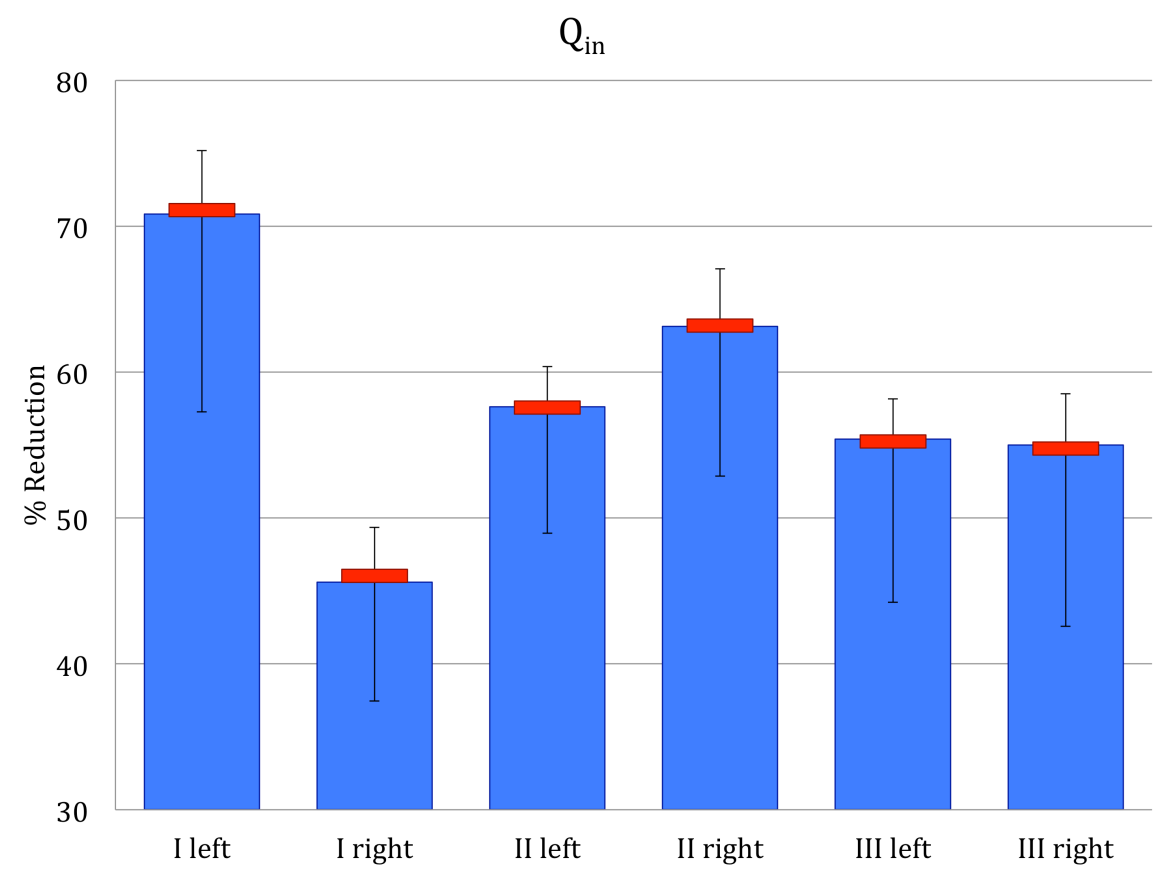

Figure 7: Aneurysm inflow reduction by geometry and device position. Steady state results are indicated in red, with the mean and range of transient results indicated in blue and black respectively

In Figure 7 substantial differences in inflow reduction, dependent on device position alone, are seen in two aneurysms: a difference of $25.3 \%$ for Aneurysm I and $5.5 \%$ for Aneurysm II. No significant difference $(<1 \%)$ in inflow reduction is seen in Aneurysm III. It is particularly striking that with a device deployed in the 
left and right daughter vessels respectively, Aneurysm I offers both the highest and lowest inflow reductions seen across all the simulation results.

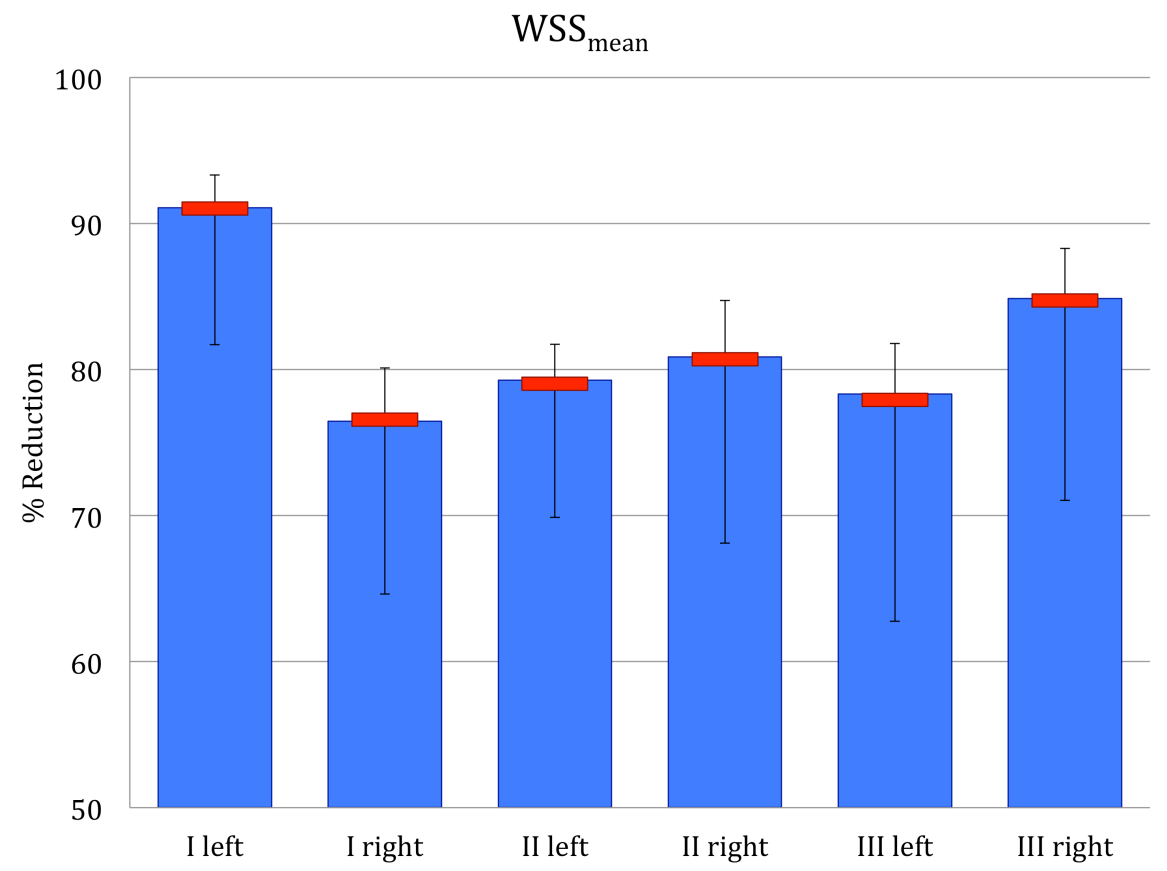

Figure 8: Aneurysm mean WSS reduction by geometry and device position. Steady state results are indicated in red, with the mean and range of transient results indicated in blue and black respectively 
Although less variation is seen in mean-WSS reduction across the aneurysm and device combinations (shown in Figure 8), the differences of $14.6 \%$ and $6.5 \%$, for Aneurysms I and III respectively are still notable.

The corresponding differences in maximum-WSS reduction (Figure 9) are more modest still, at 5.7\%, 3.3\% and $12.1 \%$ for Aneurysms I, II and III respectively, but an emerging pattern of a more successful device placement position in each aneurysm is further reinforced.

Overall the percentage reduction in both measures of WSS is consistently higher ( $>75 \%$ in all cases) than the reduction in aneurysm inflow achieved, which ranges from approximately $45 \%$ to $70 \%$. Across all three aneurysms, one configuration of device placement appears to offer better performance in each case: the left configuration for Aneurysm I, and the right configuration for Aneurysms II and III. In each case (excluding Aneurysm III $Q_{\text {in }}$ and Aneurysm II WSS $_{\text {mean }}$ ), this preferential configuration significantly reduces each metric examined. As such, these preferential device positions may then translate to an increased probability of aneurysm thrombosis and successful treatment outcome, although such an assertion is currently unverified. 


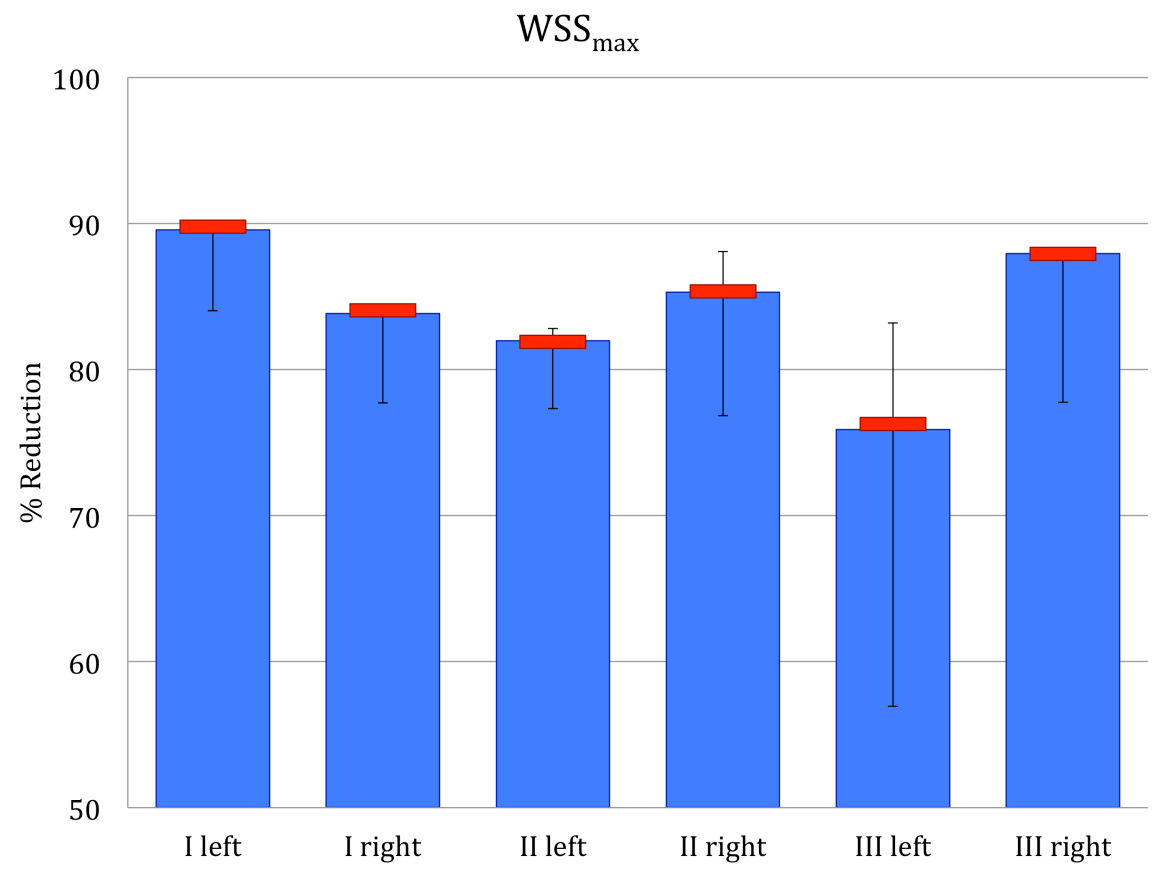

Figure 9: Aneurysm maximum WSS reduction by geometry and device position.

Steady state results are indicated in red, with the mean and range of transient results indicated in blue and black respectively

Across all three aneurysm geometries, both with and without a device deployed, one of the two daughter vessels considered is dominant and transports a larger overall fraction of the parent vessel flow rate, as shown in Table 1. The variation due to device positioning in the proportion of flow exiting each daughter vessel is 
very small $(<2 \%$ of the total flow rate in all cases). Interestingly, the preferential device configuration for both Aneurysms I and III corresponds to deploying the flow-diverter device in the non-dominant daughter vessel with a lower overall fraction of outflow.

Table 1: Percentages of total mean flow exiting each daughter vessel with No Device deployed and by deployed device configuration (Left and Right). The relative increase or decrease in vessel flow rate from the No Device case is also indicated in brackets.

\begin{tabular}{|c|c|c|c|c|c|c|c|c|c|}
\cline { 2 - 9 } \multicolumn{1}{c|}{} & \multicolumn{4}{c|}{ No Device } & \multicolumn{3}{c|}{ Left } & \multicolumn{3}{c|}{ Right } \\
\cline { 2 - 10 } \multicolumn{1}{c|}{} & Left & Right & Others & Left & Right & Others & Left & Right & Others \\
\hline Aneurysm I & 32.0 & 40.0 & 28.0 & $\begin{array}{c}34.8 \\
(+8.75)\end{array}$ & $\begin{array}{c}41.3 \\
(+3.25)\end{array}$ & $\begin{array}{c}23.9 \\
(-14.6)\end{array}$ & $\begin{array}{c}34.3 \\
(+7.19)\end{array}$ & $\begin{array}{c}41.2 \\
(+3.00)\end{array}$ & $\begin{array}{c}24.5 \\
(-12.5)\end{array}$ \\
\hline Aneurysm II & 31.2 & 43.6 & 25.2 & $\begin{array}{c}30.9 \\
(-0.96)\end{array}$ & $\begin{array}{c}43.5 \\
(-0.23)\end{array}$ & $\begin{array}{c}25.6 \\
(+1.59)\end{array}$ & $\begin{array}{c}31.8 \\
(+1.92)\end{array}$ & $\begin{array}{c}44.8 \\
(+2.75)\end{array}$ & $\begin{array}{c}23.4 \\
(-7.14)\end{array}$ \\
\hline Aneurysm III & 46.5 & 35.8 & 17.7 & $\begin{array}{c}47.9 \\
(+3.01)\end{array}$ & $\begin{array}{c}36.7 \\
(+2.51)\end{array}$ & $\begin{array}{c}15.4 \\
(-13.0)\end{array}$ & $\begin{array}{c}48.3 \\
(+3.87)\end{array}$ & $\begin{array}{c}34.9 \\
(-2.51)\end{array}$ & $\begin{array}{c}16.8 \\
(-5.08)\end{array}$ \\
\hline
\end{tabular}

Figures 10 and 11 detail the streamlines and wall shear stress distributions for each aneurysm and begin to illustrate some underlying mechanisms behind the variation due to device positioning seen in Figures 7, 8 and 9. The details of these distributions are discussed on a case-by-case basis below: 


\section{Aneurysm I}

The large variation in aneurysm inflow reduction for the Aneurysm I geometry, seen in Figure 7, is also clearly visible in the streamlines of Figure 10. For Aneurysm I with a device deployed in either daughter vessel, blood enters on the right-hand side of the aneurysm but then exits in the direction corresponding to the un-stented daughter vessel. Consequently, flow is both entering and exiting the aneurysm in the same region in the Left configuration, which appears to substantially reduce the degree to which higher speed $\left(\sim 0.25 \mathrm{~ms}^{-1}\right)$ flow penetrates the aneurysm dome. By contrast, in the Right device configuration almost all flow enters the aneurysm dome on the right, circulates throughout the entire aneurysm dome and then exits through the un-stented left daughter vessel.

The variation in $Q_{\text {in }}$ reduction seen between device configurations for Aneurysm I may be due to difference in apparent porosity of the device, which is dependent upon both the degree of device opening and the incident angle at which flow strikes the device mesh. In the Left configuration, flow at the aneurysm neck appears to strike the device at a shallower angle than for the Right, thereby increasing the device's relative porosity. Similarly, in the Right case, the wider 
ostium of the right-hand daughter vessel may result in greater device opening and lower device porosity locally, precisely where significant aneurysm inflow occurs.

However, the inflow reduction seen for Aneurysm I does not appear to be explained by porosity alone. The proportion of overall flow in each stented daughter vessel, $34.8 \%$ and $41.2 \%$ respectively for the Left and Right cases, implies that more flow passes through the device mesh in the Left case: $65.3 \%$ vs $58.8 \%$. But, a lower proportion of the flow passing through the device then enters aneurysm dome in the Left case, a phenomenon that appears to be dictated by the aneurysm geometry and overall flow environment. Overall, Aneurysm I has the least symmetric aneurysm dome and, correspondingly, the least-symmetric aneurysm flow pattern. Although not included in the figure, a similar flow pattern to the Right configuration was observed when no device was deployed in the aneurysm geometry. 


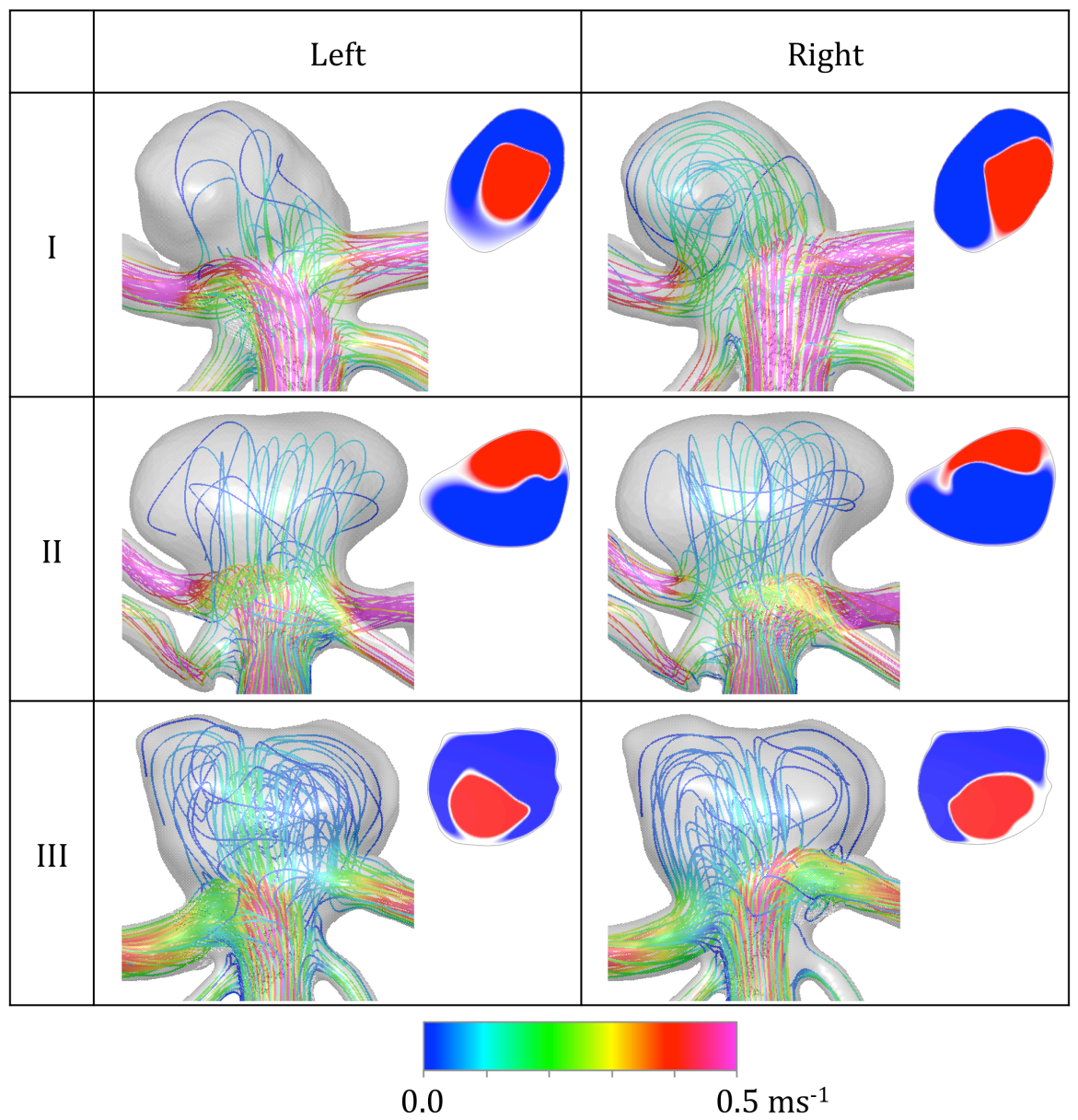

Figure 10: Flow streamlines for transient simulations shown at mean flow rate by geometry and device position. Inflow (red) and outflow (blue) regions of the aneurysm inlet plane are also shown

The WSS distributions in Aneurysm I follow a similar pattern to the flow streamlines for the corresponding device positions. For mean-WSS value, the Left 
and Right device configurations again represent the highest and lowest reductions respectively seen across all aneurysms. As shown in Figure 11, there are significant spatial differences in the aneurysm WSS distributions for the Left and Right configurations. This difference is most stark on the front face of the aneurysm dome (left-hand image of each pair), where in the Left configuration local flow stasis has reduced the WSS to very near zero across the entire surface. Overall, in both deployment positions, the device reduces the entire aneurysm WSS distribution to a magnitude considered physiologically normal.

\section{Aneurysm II}

The inflow patterns of Aneurysm II are very similar across the Left and Right configurations, as shown in Figure 10. In both cases higher-velocity flow enters in the neck region towards the back-face of the aneurysm, and this flow then circulates through the entire aneurysm dome as a single vortex before exiting along the front edge of the aneurysm neck. In each case the majority of the blood leaving the aneurysm appears to do so in the direction of the corresponding unstented daughter vessel. 
The angle at which flow strikes the device mesh and the daughter vessel ostium, and hence the effective device porosity, appear similar for both device configurations. Thus, the relatively small 5.5\% difference in $Q_{\text {in }}$ reduction seen between the Left and Right configurations may not be the result of porosity differences, but the different fractions of flow exiting via each daughter vessel alone. As shown in Table 1, a larger fraction of the overall flow passes through the device mesh in the Left compared to the Right configurations (69.1\% vs 55.2\%). Hence, given the very similar overall flow regimes between the two configurations, it seems logical more flow would then enter the aneurysm overall in the Left configuration and thereby reduce the device's $Q_{\text {in }}$ reduction.

The WSS distributions for each deployed device position also appear almost identical, which correlates with the insignificant different in mean WSS reduction detailed in Figure 8. Although slightly elevated areas of WSS are common to both device configurations, in each case the elevated areas are shifted marginally away from the stented daughter vessel, especially at the aneurysm neck. These areas of higher WSS occur in regions where flow exits the aneurysm dome via the unstented daughter vessel. In both the Left and Right device configurations, peak WSS values are located in these regions and adjacent to the un-stented daughter vessel 
in each case. As in the case of Aneurysm I, both device positions reduce the WSS in the entirety of the aneurysm dome to values considered physiologically normal.

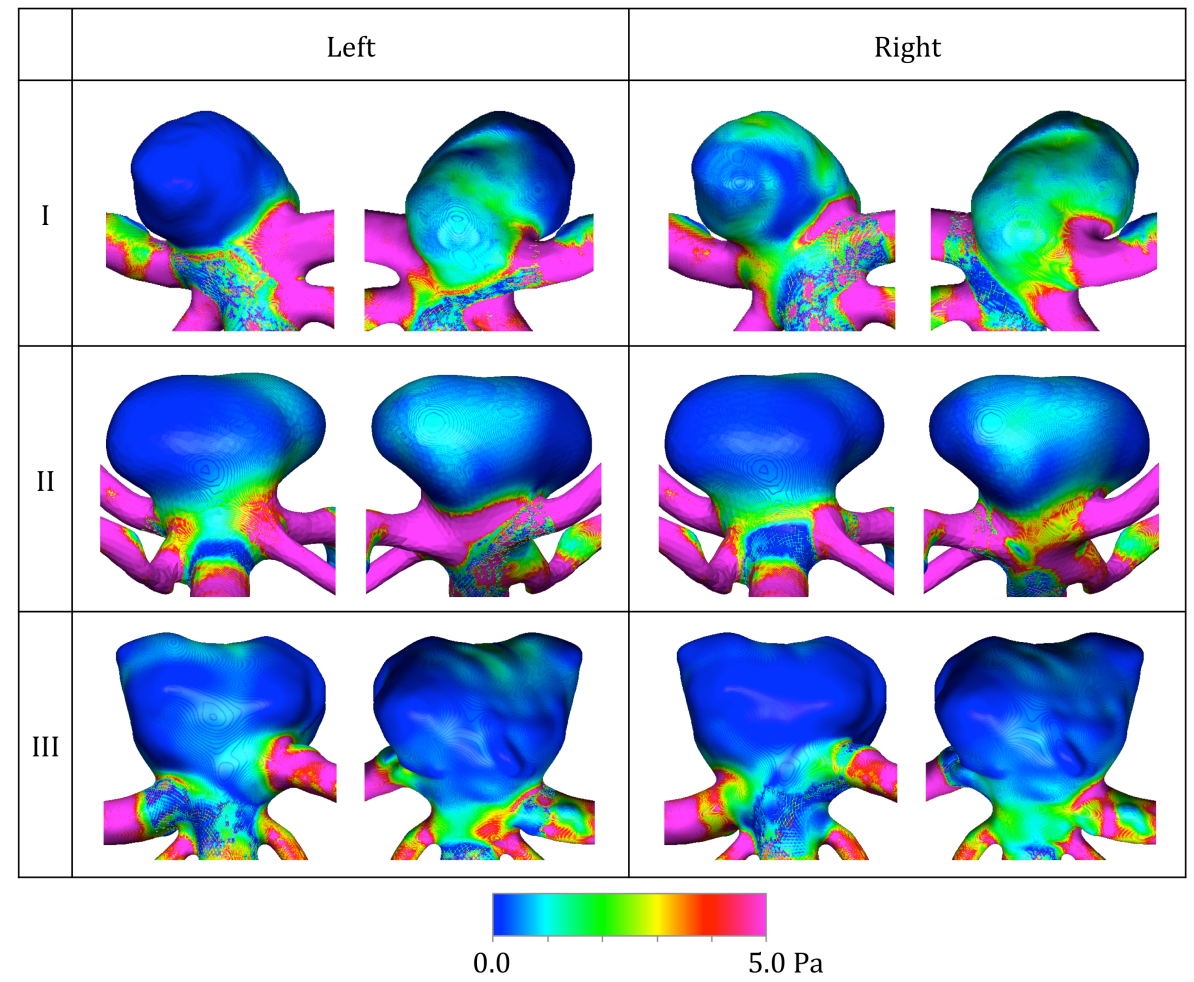

Figure 11: WSS distributions for transient simulations shown at mean flow rate by geometry and device position (front and back views) 


\section{Aneurysm III}

For Aneurysm III the inflow streamlines are also similar regardless of device positioning, as shown in Figure 10. Broadly, flow enters the aneurysm in the frontcentral portion of the neck and circulates towards the back face in two vortices that are aligned with the lobes of the aneurysm dome. A very similar flow pattern was also seen in the No Device case, which is not included in the figure for brevity.

Again, flow appears to exit the aneurysm primarily in the direction of the unstented vessel. Although more of the total flow passes through the device mesh in the Right configuration (65.1\% vs. 52.1\%) a significant fraction of the flow moves directly into the un-stented (left) daughter vessel in this case. By contrast, the flow enters the un-stented (right) daughter vessel in the Left configuration almost entirely via the aneurysm dome. Hence, as in the case of Aneurysm I, although more flow passes through the device mesh in the preferential configuration, the local flow environment results in a smaller fraction of this flow entering the aneurysm dome. 
Despite a very similar inflow reduction between the Left and Right configurations, the flow entering the Left geometry does so with a more pronounced jet, which penetrates further into the aneurysm geometry (see Figure 10). This more coherent jet structure results in locally increased WSS due to impact at the tip of the aneurysm in the Left configuration, clearly visible in Figure 11. The more favourable reductions in both mean and maximum WSS achieved by the device deployed in the Right configuration appear to be a direct result of more effective suppression of this inflow jet. For Aneurysm III, as in all other cases, the deployment of a flow-diverter in either daughter vessel reduces the WSS distribution in the aneurysm dome to a range considered physiologically normal.

Across all three aneurysm geometries an increase in total flow delivered to both daughter vessels considered (Left and Right) occurs following device deployment in either configuration in all but one case: Aneurysm II Left. It appears in the remaining five cases flow to the other two daughter vessels (LS and RS in Figure 1 and referred to collectively as "Others" in Table 1) is reduced by 5.08-14.6\%. These "Others" vessels are jailed in all device configurations and are both smaller in diameter than the Left and Right vessels, and are orientated at a more obtuse angle to the flow direction - factors that appear to exacerbate the effects of jailing. By 
contrast, there does not appear to be a correlation between placement of a device in a vessel and a corresponding increase in vessel flow rate for the Left and Right vessels.

Finally, a summary of the simulation workflow completed is detailed in Table 2, with estimates of the corresponding execution time for each stage of the virtual treatment pipeline. A complete virtual treatment case (with and without a device deployed) may be simulated in less than 5 hours, with a steady state calculation and an assumption of a solution mesh independence to the level discussed. Comparing two devices or two positions of the same device would require around a further 2.5 hours. 
Table 2: Simulation workflow with hardware and software setups and corresponding execution times. ${ }^{1}$ Assuming a mesh size equivalent to the level of mesh-independence previously detailed

\begin{tabular}{|c|c|c|c|}
\hline Process & Hardware & Software & $\begin{array}{c}\text { Approximate } \\
\text { Execution Time } \\
\text { (hrs) }\end{array}$ \\
\hline $\begin{array}{c}\text { Segmentation/ } \\
\text { Pre-Processing }\end{array}$ & $\begin{array}{c}1 \times 2.66 \mathrm{GHz} \text { CPU } \\
4 \mathrm{~GB} \mathrm{RAM}\end{array}$ & $\begin{array}{c}\text { OsiriX } \\
\text { (mac) }\end{array}$ & 0.25 \\
\hline Device Deployment & $\begin{array}{c}1 \times 2.66 \mathrm{GHz} \text { CPU } \\
4 \mathrm{~GB} \mathrm{RAM}\end{array}$ & $\begin{array}{c}\text { Matlab } \\
\text { (mac) }\end{array}$ & 0.1 \\
\hline Mesh Generation 1 & $\begin{array}{c}16 \times 2.40 \mathrm{GHz} \text { CPUs } \\
32 \mathrm{~GB} \mathrm{RAM}\end{array}$ & $\begin{array}{c}\text { CFD-VisCART } \\
\text { (windows) }\end{array}$ & 0.5 \\
\hline CFD Solver 1 & $\begin{array}{c}32 \times 2.80 \mathrm{GHz} \text { CPUs } \\
128 \mathrm{~GB} \text { RAM }\end{array}$ & $\begin{array}{c}\text { CFD-ACE+ } \\
\text { (linux) }\end{array}$ & $\begin{array}{c}1.5 \text { (steady) } \\
75 \text { (transient) }\end{array}$ \\
\hline Post-Processing & $\begin{array}{c}16 \times 2.40 \mathrm{GHz} \text { CPUs } \\
32 \mathrm{~GB} \text { RAM }\end{array}$ & $\begin{array}{c}\text { CFD-VIEW } \\
\text { (windows) }\end{array}$ & 0.25 \\
\hline
\end{tabular}

The surprising, and perhaps counter-intuitive, flow patterns induced by different device positions that have been elucidated in this study illustrate that optimal flow-diverter placement is far from trivial. With the computational framework proposed, accurate patient-specific simulations of multiple treatment options would be feasible in the clinical setting with overnight computations on highperformance desktop hardware. 


\section{Study Limitations}

The results of this computational study should be viewed in the context of a number of limitations. The small quantity of aneurysm geometries simulated immediately limits the generalisability of the study conclusions, and it is unclear whether the findings presented are in any way representative. The use of average flow profiles and flow rates in addition to uniform boundary conditions at all outlets may be unrealistic and certainly reduces the patient-specific nature of the simulations. In particular, the assumption of symmetric outlet boundary conditions may be unrealistic, given the subtle asymmetric flow patterns observed across all simulated aneurysm geometries. Finally, as discussed in the introduction, the flow metrics reported in this study are considered surrogate measures that are linked with aneurysm thrombosis. However, the precise factors governing aneurysm treatment outcome remain poorly understood, rendering any predictions of significant difference in treatment outcome due to device position entirely unverified.

\section{Conclusions}

In this study of virtual aneurysm treatment, substantial differences due to flowdiverter device position alone were observed for a number of haemodynamic 
metrics often linked with positive clinical outcomes. In all cases, the flow environment in and around the aneurysm was shown to be complex and nontrivial. In two-thirds of the aneurysms the greatest reduction in the metrics was achieved when a flow-diverter device was deployed in the non-dominant daughter vessel with a lower overall flow rate. Relatively subtle differences in overall aneurysm morphology, and particularly the degree of aneurysm symmetry, were shown to result in substantial changes in haemodynamic environment following device deployment. Although device jailing appears to reduce the flow rate in smaller daughter vessels that were angled away from the flow direction, flow in larger vessels appeared relatively unaffected by the device.

A larger study of the same virtual treatment procedure has the potential to elucidate haemodynamic mechanisms underlying the high failure-rate of bifurcation aneurysms treated by flow-diverter that are detailed in the literature. For the aneurysms discussed, combining steady state CFD simulations with simplified device deployment techniques results in a computational load sufficiently modest enough to make such predictions a possibility in the clinical setting. 


\section{Acknowledgements}

The authors would like to thank Dr M. Megahed and the ESI Group for allowing the use of the CFD-ACE suite, and also acknowledge the support of the Engineering and Physical Sciences Research Council (grant number: NS/A000008/1). 


\section{References}

1. Byrne J V, Szikora I. Flow Diverters in the Management of Intracranial Aneurysms : A Review. EJMINT. 2012;

2. Alfano J, Kolgega J, Natarajan SK, Xiang J, Paluch R, Levy E, et al. Intracranial Aneurysms Occur More Frequently at Bifurcation Sites that Typically Experience Higher Hemodynamic Stresses. Neurosurgery. 2013;

3. Brinjikji W, Cloft HJ, Fiorella D, Lanzino G, Kallmes DF. Estimating the proportion of intracranial aneurysms likely to be amenable to treatment with the pipeline embolization device. J Neurointerv Surg [Internet]. 2011 Dec 2 [cited 2012 Jul 19];3-6. Available from: http://www.ncbi.nlm.nih.gov/pubmed/22140137

4. Saatchi I, Yavuz K, Ozer C, Geyik S, Cekirge HS. Treatment of Intracranial Aneurysms Using the Pipeline Flow-Diverter Embolization Device: A SingleCenter Experience with Long-Term Follow- Up Results. AJNR. 2012;33.

5. Castro M, Putman C, Radaelli A, Frangi A, Cebral J. Hemodynamics and Rupture of Terminal Cerebral Aneurysms. Acad Radiol. 2009;16:1201-7.

6. Tang AY, Eric WC, Anderson JQ, Gilberto CT, Alfred KL, Chow CYK. Computational Fluid Dynamics Study of Bifurcation Aneurysms Treated with 
Pipeline Embolization Device : Side Branch Diameter Study. J Med Biol Eng [Internet]. Springer Berlin Heidelberg; 2015;35(3):293-304. Available from: "http://dx.doi.org/10.1007/s40846-015-0046-3

7. Fernandez H, Macho JM, Blasco J, Roman LS, Mailaender W, Serra L, et al. Computation of the change in length of a braided device when deployed in realistic vessel models. Int J Comput Assist Radiol Surg [Internet]. 2015; Available from: http://link.springer.com/10.1007/s11548-015-1230-1

8. Aurboonyawat T, Blanc R, Schmidt P, Piotin M, Spelle L, Nakib A, et al. An in vitro study of silk stent morphology. Neuroradiology. 2011;53(9):659-67.

9. Saleme S, Iosif C, Ponomarjova S, Mendes G, Camilleri Y, Caire F, et al. FlowDiverting Stents for Intracranial Bifurcation Aneurysm Treatment. Neurosurgery [Internet]. 2014;75(6):623-31. Available from: http://content.wkhealth.com/linkback/openurl?sid=WKPTLP:landingpage\& an $=00006123-201412000-00013$

10. Caroff J, Neki H, Mihalea C, D’Argento F, Abdel Khalek H, Ikka L, et al. FlowDiverter Stents for the Treatment of Saccular Middle Cerebral Artery Bifurcation Aneurysms. AJNR Am J Neuroradiol [Internet]. 2015;1-6. Available from: http://www.ncbi.nlm.nih.gov/pubmed/26405085 
11. Fahed, R; Gentric, JC; Salazkin, I; Gevry, G; Raymond, J; Darsaut T. Flow diversion of bifurcation aneurysm is more effective when the jailed branch is occluded: an experimental study in a novel canine model. J NeurInterventional Surg. 2016;sous press:1-5.

12. Kulcsár Z, Ernemann U, Wetzel SG, Bock A, Goericke S, Panagiotopoulos V, et al. High-profile Flow Diverter (Silk) Implantation in the Basilar Artery. Stroke [Internet]. 2010 Aug [cited 2011 Aug 5];41(8):1690-6. Available from: http://www.ncbi.nlm.nih.gov/pubmed/20616327

13. Wong GKC, Kwan MCL, Ng RYT, Yu SCH, Poon WS. Flow diverters for treatment of intracranial aneurysms: Current status and ongoing clinical trials. J Clin Neurosci. 2011;18(6):737-40.

14. Mangiafico S, Guarnieri G, Consoli A, Ambrosanio G, Muto M. Endovascular Strategy for Unruptured Cerebral aneurysms. Eur J Radiol. 2012;

15. Iosif C, Mounayer C. Flow-Diverter Stents for the Treatment of Saccular Middle Cerebral Artery Bifurcation Aneurysms: Is "Unsuitable” the Right Conclusion? AJNR Am J Neuroradiol [Internet]. 2016;37:13. Available from: http://www.ncbi.nlm.nih.gov/pubmed/26405085

16. Ngoepe MN, Ventikos Y. Computational modelling of clot development in 
patient- specific cerebral aneurysm cases. J Thromb Haemost [Internet]. 2016;14(2):262-72. Available from:

http://doi.wiley.com/10.1111/jth.13220

17. Cito S, Mazzeo MD, Badimon L. A review of macroscopic thrombus modeling methods. Thromb Res [Internet]. 2013 Feb;131(2):116-24. Available from: http://www.ncbi.nlm.nih.gov/pubmed/23260443

18. Furie B, Furie BC. Mechanisms of thrombus formation. N Engl J Med [Internet]. 2008 Aug 28;359(9):938-49. Available from: http://www.ncbi.nlm.nih.gov/pubmed/18753650

19. Cebral JR, Mut F, Raschi M, Hodis S, Ding Y-H, Erickson BJ, et al. Analysis of Hemodynamics and Aneurysm Occlusion after Flow-Diverting Treatment in Rabbit Models. AJNR Am J Neuroradiol [Internet]. 2014; Available from: http://www.ncbi.nlm.nih.gov/pubmed/24722302

20. Byrne J V, Hubbard N. A Novel Two-Stage Technique for Construction of Experimental Aneurysms. Ajnr Am J Neuroradiol. 2004;(February):319-21.

21. Gester K, Lu I, Bu M. In Vitro Evaluation of Intra-Aneurysmal , Flow-DiverterInduced Thrombus Formation: A Feasibility Study. AJNR. 2016;1-7.

22. Cebral JR, Vazquez M, Sforza DM, Houzeaux G, Tateshima S, Scrivano E, et al. 
Analysis of hemodynamics and wall mechanics at sites of cerebral aneurysm rupture. J Neurointerv Surg [Internet]. 2014;1-7. Available from: http://www.ncbi.nlm.nih.gov/pubmed/24827066

23. Chen H, Selimovic A, Thompson H, Chiarini A, Penrose J, Ventikos Y, et al. Investigating the Influence of Haemodynamic Stimuli on Intracranial Aneurysm Inception. Ann Biomed Eng [Internet]. 2013 Jul [cited 2014 Mar 27];41(7):1492-504. Available from:

http://www.ncbi.nlm.nih.gov/pubmed/23553330

24. Griffith TM. Modulation of Blood Flow and Tissue Perfusion by EndotheliumDerived Relaxing Factor. Exp Physiol. 1994;79:873-913.

25. Peach TW, Ngoepe M, Spranger K, Zajarias-Fainsod D, Ventikos Y. Personalizing flow-diverter intervention for cerebral aneurysms: from computational hemodynamics to biochemical modeling. Int j numer method biomed eng [Internet]. 2014 Nov;30(11):1387-407. Available from: http://www.ncbi.nlm.nih.gov/pubmed/25045060

26. Ouared R, Chopard B, Stahl B, Rüfenacht D a., Yilmaz H, Courbebaisse G. Thrombosis modeling in intracranial aneurysms: a lattice Boltzmann numerical algorithm. Comput Phys Commun [Internet]. 2008 Jul [cited 2013 Aug 1];179(1-3):128-31. Available from: 
http://linkinghub.elsevier.com/retrieve/pii/S0010465508000283

27. Chong W, Zhang Y, Qian Y, Lai L, Parker G, Mitchell K. Computational hemodynamics analysis of intracranial aneurysms treated with flow diverters: Correlation with clinical outcomes. Am J Neuroradiol. 2014;35(1):136-42.

28. Ribeiro de Sousa D, Vallecilla C, Chodzynski K, Corredor Jerez R, Malaspinas O, Eker OF, et al. Determination of a shear rate threshold for thrombus formation in intracranial aneurysms. J Neurointerv Surg [Internet]. 2015;(August):neurintsurg - 2015-011737. Available from: http://jnis.bmj.com/lookup/doi/10.1136/neurintsurg-2015-011737

29. Malaspinas O, Turjman A, Ribeiro de Sousa D, Garcia-Cardena G, Raes M, Nguyen PTT, et al. A spatio-temporal model for spontaneous thrombus formation in cerebral aneurysms. J Theor Biol. 2016;394:68-76.

30. Spranger K, Ventikos Y. Which spring is the best? Comparison of methods for virtual stenting. IEEE Trans Biomed Eng [Internet]. 2014 Jul;61(7):19982010. Available from: http://www.ncbi.nlm.nih.gov/pubmed/24956618

31. Ni M, Abdou MA. A Bridge Between Projection Methods and SIMPLE Type Methods for Incompressible Navier - Stokes Equations. IJNMBE. 
2007;72:1490-512.

32. Doormaal JP Van, Raithby GD. Enhancements of the Simple Method for

Predicting Incompressible Fluid Flows. Numer Heat Transf. 1984;7:147-63.

33. Webster R. An Algebraic Multigrid Solver for Navier-Stokes Problems. Int J Numer Methods Fluids. 1994;18:761-80.

34. Perktold K, Resch M, Florian H. Pulsatile Non-Newtonian Flow Characteristics in a Three-dimensional Human Carotid Bifurcation Model. Biomech Eng [Internet]. 1991 Nov;113(4):464-75. Available from: http://www.ncbi.nlm.nih.gov/pubmed/1762445

35. Valencia A a, Guzmán AM, Finol E a, Amon CH. Blood Flow Dynamics in Saccular Aneurysm Models of the Basilar Artery. Biomech Eng [Internet]. 2006 Aug;128(4):516-26. Available from:

http://www.ncbi.nlm.nih.gov/pubmed/16813443

36. Dempere-Marco L, Oubel E, Castro M, Putman C, Frangi A, Cebral J. CFD Analysis Incorporating the Influence of Wall Motion: Application to Intracranial Aneurysms. MICCAI 2006 [Internet]. 2006 Jan;438-45. Available from: http://www.ncbi.nlm.nih.gov/pubmed/17354802 